\title{
Nuclear localisation of nuclear factor-kappaB transcription factors in prostate cancer: an immunohistochemical study
}

\author{
L Lessard', LR Bégin ${ }^{2}$, ME Gleave ${ }^{3}$, A-M Mes-Masson ${ }^{1,4}$ and F Saad*,1,5 \\ 'Centre de recherche du CHUM, and Institut du cancer de Montréal, 1560 Sherbrooke Est, Montréal, Québec, Canada H2L 4MI; ${ }^{2}$ Service \\ d'anatomopathologie, Hôpital du Sacré-Cœur de Montréal, 5400 boul. Gouin Ouest, Montréal, Québec, Canada H4J IC5; ${ }^{3}$ The Prostate Centre, \\ Vancouver General Hospital, University of British Columbia, D-9, 2733 Heather Street, Vancouver, British Columbia, Canada V5Z 3/5; ${ }^{4}$ Département de \\ médecine, Université de Montréal, Québec, Canada; ${ }^{5}$ Département de chirurgie (urologie), CHUM-Notre-Dame, 1560 Sherbrooke Est, Montréal, \\ Québec, Canada H2L 4MI
}

\begin{abstract}
Several reports suggest that the canonical nuclear factor-kappaB (NF- $\kappa$ B) pathway is constitutively activated in a subset of prostate cancer cells. However, except for RelA (p65), little is known about the status of NF- $\kappa$ B transcription factors in prostate cancer tissues. To clarify the status of NF- $\kappa$ B subunits, we analysed the expression and subcellular localisation of RelA, RelB, c-Rel, p50, and p52 on tissue array sections containing respectively 344, 346, 369, 343, and 344 cores from 75 patients. The subcellular localisation of NF- $\kappa$ B factors was tested against relevant clinical parameters (preoperative prostate-specific antigen, pathological stage, and postoperative Gleason grade). With the exception of c-Rel, each subunit was detected in the nucleus of cancer cells: significant nuclear expression of RelB, RelA, p52, and p50 was seen in 26.6, 15.6, 10.7, and 10.5\% of cores, respectively. Surprisingly, cores expressing both nuclear RelA and p50 canonical pathway proteins were less frequently observed than cores expressing other subunit combinations such as RelB-p52 and RelA-RelB. In addition, the nuclear localisation of RelB correlated with patient's Gleason scores (Spearman correlation: $0.167 ; P=0.018$ ). The nuclear localisation of both canonical and noncanonical NF- $\kappa \mathrm{B}$ subunits in prostate cancer cells suggests for the first time that different NF- $\kappa$ B pathways and dimers may be activated in the progression of the disease.

British Journal of Cancer (2005) 93, 1019 - 1023. doi:I0.1038/sj.bjc.6602796 www.bjcancer.com

Published online 4 October 2005

(c) 2005 Cancer Research UK
\end{abstract}

Keywords: prostate cancer; NF- $\kappa B$; immunohistochemistry; tissue microarray; Gleason grade

In Caucasian men, prostate cancer is the most frequently diagnosed cancer and a leading cause of cancer death. Presently, the majority of clinically localised tumours are detected early and treated aggressively even though some early-stage tumours can remain latent and may not require aggressive therapy. New prognostic tools are needed to distinguish between low- and highrisk tumours in order to tailor treatment strategies. Understanding the molecular mechanisms of prostate cancer progression will undoubtedly lead to the development of new molecular prognostic markers.

The nuclear factor-kappaB (NF- $\kappa \mathrm{B})$ transcription factor family is composed of five structurally related members that possess an N-terminal Rel homology (RH) domain involved in proteinprotein interactions and DNA binding (Ghosh and Karin, 2002). The NF- $\kappa \mathrm{B}$ proteins can be divided into two classes based on sequences $\mathrm{C}$-terminal to their $\mathrm{RH}$ domain. The class 1 includes Rel (c-Rel), RelA (p65), and RelB proteins that are characterised by a C-terminal transactivation domain. NF- $\kappa \mathrm{B} 1$ (p50 and its precursor p105) and NF- $\kappa$ B2 (p52 and its precursor p100) form the second

\footnotetext{
* Correspondence: Dr F Saad, Département d'urologie, Centre Hospitalier de l'Université de Montréal - Hôpital Notre-Dame, 1560 rue Sherbrooke E, Montréal, Québec, Canada H2L 4MI;

E-mail: Fred.Saad.CHUM@ssss.gouv.qc.ca

Received 5 May 2005; revised 9 August 2005; accepted 23 August 2005; published online 4 October 2005
}

class; p105 and p100 contain inhibitory C-terminal ankyrin repeats that are cleaved to create transcriptionally active p50 and p52 proteins. Rel/NF- $\kappa \mathrm{B}$ proteins exist as homo- and heterodimers. The best-studied dimers include the canonical (RelA/p50) and noncanonical (RelB/p52) NF- $\kappa$ B complexes. Normally, the activation of NF- $\kappa \mathrm{B}$ requires signals that converge to I $\kappa \mathrm{B}$ kinases (IKKs). In the canonical pathway, the IKK complex (IKK $\alpha, \beta, \gamma)$ phosphorylates $\mathrm{I} \kappa \mathrm{Bs}$, which are then ubiquitinated and targeted for proteasome-dependent degradation. In the noncanonical pathway, IKK $\alpha$ regulates the processing of the p100 precursor. As a result of the activation of either pathway, NF- $\kappa$ B dimers translocate to the nucleus and activate the expression of various genes involved in cell growth, differentiation, inflammatory responses, and the regulation of apoptosis (Aggarwal, 2004).

The NF- $\kappa \mathrm{B}$ pathways and genes are frequently altered in lymphoid and nonlymphoid cancers (Rayet and Gelinas, 1999). For instance, chromosomal alterations involving the c-Rel and $N F-\kappa B 2$ genes have been detected in several B- and T-cell lymphomas (Liptay et al, 1997; Munzert et al, 2000). It has been shown that constitutively nuclear and active RelA/p50 dimers can prevent cell death by apoptosis in many cancer cell types after chemotherapy, radiotherapy, or TNF- $\alpha$ treatment (Barkett and Gilmore, 1999; Baldwin, 2001) and have also been detected in the nuclei of pancreatic and breast carcinomas (Sovak et al, 1997; Wang et al, 1999). More recently, constitutive activation of NF- $\kappa$ B (RelA/p50) has been detected in androgen-independent prostate cancer cell lines as well as in prostate cancer tissues (Chen and Sawyers, 2002; 
Gasparian et al, 2002; Suh et al, 2002; Ayala et al, 2004; Ross et al, 2004; Shukla et al, 2004; Sweeney et al, 2004) and appears to promote cell growth, survival, and metastasis (Huang et al, 2001; Hodge et al, 2003; Levine et al, 2003; Ling et al, 2003). In particular, we and others have shown that nuclear localisation of RelA in primary prostate tumours is linked to poor clinical outcomes (Lessard et al, 2003; Ayala et al, 2004; Fradet et al, 2004; Ismail et al, 2004; Ross et al, 2004).

While RelA has become a candidate prognostic marker of prostate cancer progression, little is known about the expression and the subcellular localisation of other NF- $\kappa$ B subunits. The present study provides the first large-scale immunohistochemical characterisation of all NF- $\kappa \mathrm{B}$ transcription factors in tissuearrayed prostate cancer specimens. Our results show that all NF- $\kappa \mathrm{B}$ subunits are expressed in prostate tissues and that except for c-Rel, they are often detected in the nucleus of cancer cells. Interestingly, nuclear RelA and p50 are less frequently detected in the same cores than all other subunit combinations. In addition, the number of nuclear RelB-positive cores increased significantly with increasing Gleason scores. These results suggest that most $\mathrm{NF}-\kappa \mathrm{B}$ transcription factors, especially noncanonical subunits, may play a role in the progression of prostate cancer.

\section{MATERIALS AND METHODS}

\section{Prostate tissue microarray preparation}

Archival formalin-fixed, paraffin-embedded (FFPE) human prostate tumour specimens were used to construct a human prostate tissue array of benign, high-grade prostatic intraepithelial neoplasia (PIN), and cancer (adenocarcinoma) tissue samples. The array was subdivided into normal, PIN, low Gleason $(2,2 / 3,3)$, and high Gleason $(3 / 4,4,4 / 5,5)$ cores. Benign tissue cores were obtained from transition zone biopsies of radical prostatectomy specimens. Core tissue prostatectomy specimens $(0.6 \mathrm{~mm}$ diameter) were obtained from preselected regions of individual paraffin-embedded donor blocks and precisely arrayed into a new recipient paraffin block with a tissue arrayer (Beecher Instrument, Silver Spring, MD, USA). After the block construction was completed, $5 \mu \mathrm{m}$ sections were cut with a microtome using an adhesive-coated tape sectioning system (Instrumedics, Hackensack, NJ, USA) to support the adhesion of the array elements.

\section{Immunohistochemical staining}

Tissue sections were immunostained for NF- $\kappa \mathrm{B}$ transcription factors using the biotin-streptavidin immunoperoxidase method as previously described (Fradet et al, 2004). Briefly, FFPE tissue array slides were initially deparaffinised with toluene and rehydrated through graded ethanol. Endogenous peroxidase activity was blocked with $3 \%$ hydrogen peroxide for $10 \mathrm{~min}$. An antigen retrieval technique was applied by boiling slides for $10 \mathrm{~min}$ at $95^{\circ} \mathrm{C}$ in a $0.01 \mathrm{M}$ sodium citrate buffer, $\mathrm{pH}$ 6.0. The staining of c-Rel was enhanced using $1 \mathrm{~mm}$ EDTA ( $\mathrm{pH} 8.0$ ) instead of citrate. Tissue sections were incubated with a protein blocking serum-free reagent (Dako Diagnostics, Mississauga, ON, Canada) for $15 \mathrm{~min}$ to block nonspecific binding. The NF- $\kappa \mathrm{B}$ subunit expression was studied using mouse monoclonal NF- $\kappa$ B RelA (p65) F-6, rabbit polyclonal RelB C-19, mouse monoclonal c-Rel B6, rabbit polyclonal NF- $\kappa$ B p50 NLS (all from Santa Cruz Biotechnology, Santa Cruz, CA, USA), and mouse monoclonal NF- $\kappa$ B p52 (Upstate Biotechnology, Lake Placid, NY, USA). Primary antibodies were applied at a concentration of $1: 10$ (c-Rel) or 1:50 (RelA, RelB, $\mathrm{p} 50, \mathrm{p} 52)$ in PBS and were incubated for $2 \mathrm{~h}$ at room temperature. Immune complexes were revealed using a biotin-conjugated broad-spectrum secondary antibody $(20 \mathrm{~min})$, then streptavidinperoxidase conjugate for $20 \mathrm{~min}$ (DakoCytomation, Mississauga,
ON, Canada), followed by chromogen (0.06\% 3,3-diaminobenzidine tetrahydrochloride, $0.01 \%$ hydrogen peroxide in PBS) for $5 \mathrm{~min}$. Sections were counterstained with Mayer's haematoxylin, dehydrated, and then mounted. Negative controls were performed by omitting the primary antibody.

\section{Analysis}

Damaged or nonrepresentative tissue cores (e.g., absence of epithelial tissue) were excluded from the analysis, leaving 344 (RelA), 346 (RelB), 369 (c-Rel), 343 (p52), and 344 (p50) cores from 75 patients. Cores were scored either as positive (1) or negative ( 0 ) for unequivocal brown nuclear NF- $\kappa$ B staining in at least $5 \%$ of cancer cells. Clinical data were also available in a subset of samples in which preoperative serum prostate-specific antigen (pPSA), pathologic Gleason score (sum), and pathologic stage were available for 41,47 , and 46 patients, respectively. All statistical tests were performed using SPSS, version 10 (SPSS, Chicago, IL, USA).

\section{RESULTS}

Nuclear factor-kappaB subunit expression and subcellular localisation in prostatic tissues

As shown in Figure 1, all NF- $\kappa \mathrm{B}$ subunits were expressed in prostatic tissues. Most benign glands were characterised by a strong cytoplasmic staining of the basal cell constituent and a variable cytoplasmic staining of the secretory (luminal) cells. Nuclear factor- $\kappa \mathrm{B}$ nuclear expression was usually not detected in benign tissues with the exception of a few cores in which RelB (two out of 40) and p52 (one out of 39) were localised in the nucleus of secretory cells. Prostatic intraepithelial neoplasia samples expressed weak to strong cytoplasmic levels of NF- $\kappa \mathrm{B}$ subunits and only one core (one out of 23) demonstrated RelA nuclear staining. Finally, all NF- $\kappa \mathrm{B}$ proteins were present in the cytoplasm of cancer cells and all except c-Rel were detected in the nuclei of cancer tissues at variable frequencies. Table 1 compares the frequencies of NF- $\kappa$ B nuclear staining between normal, PIN, and cancer specimens. In all cases, the frequency of positive cores was significantly higher in cancer tissues as compared to noncancerous tissues. Within cancer specimens, nuclear RelB expression was the most commonly detected followed by RelA, p52, and p50. When cancer tissues were divided into low- to intermediategrade (Gleason scores 2, 2/3, 3) and high-grade (Gleason scores $3 / 4,4,4 / 5,5)$ tumours, no statistically significant difference was observed between the two groups, but there was a trend towards increased RelB nuclear staining in high-grade specimens (Table 2).

\section{Nuclear detection of several NF- $\kappa$ B subunits in individual cores}

The NF- $\kappa \mathrm{B}$ proteins function as homo- and heterodimers. To assess the relative importance of these dimers in prostatic tissues, we calculated the proportion of cores in which both subunits of an $\mathrm{NF}-\kappa \mathrm{B}$ heterodimer were detected in the nucleus of prostatic cells (Figure 2). Importantly, although all subunit combinations were observed in cancer cores, the canonical RelA-p50 pair was less frequently detected than other subunit pairs. In particular, the proportion of cores positive for both RelA and RelB (class 1 subunits) was significantly higher than that of cores positive for RelA and p50 $(P=0.008)$.

\section{Relationship between nuclear NF- $\kappa \mathrm{B}$ and clinical parameters in prostate cancer patients}

We performed statistical analyses to identify possible correlations and/or associations between NF- $\kappa \mathrm{B}$ nuclear staining and pPSA 


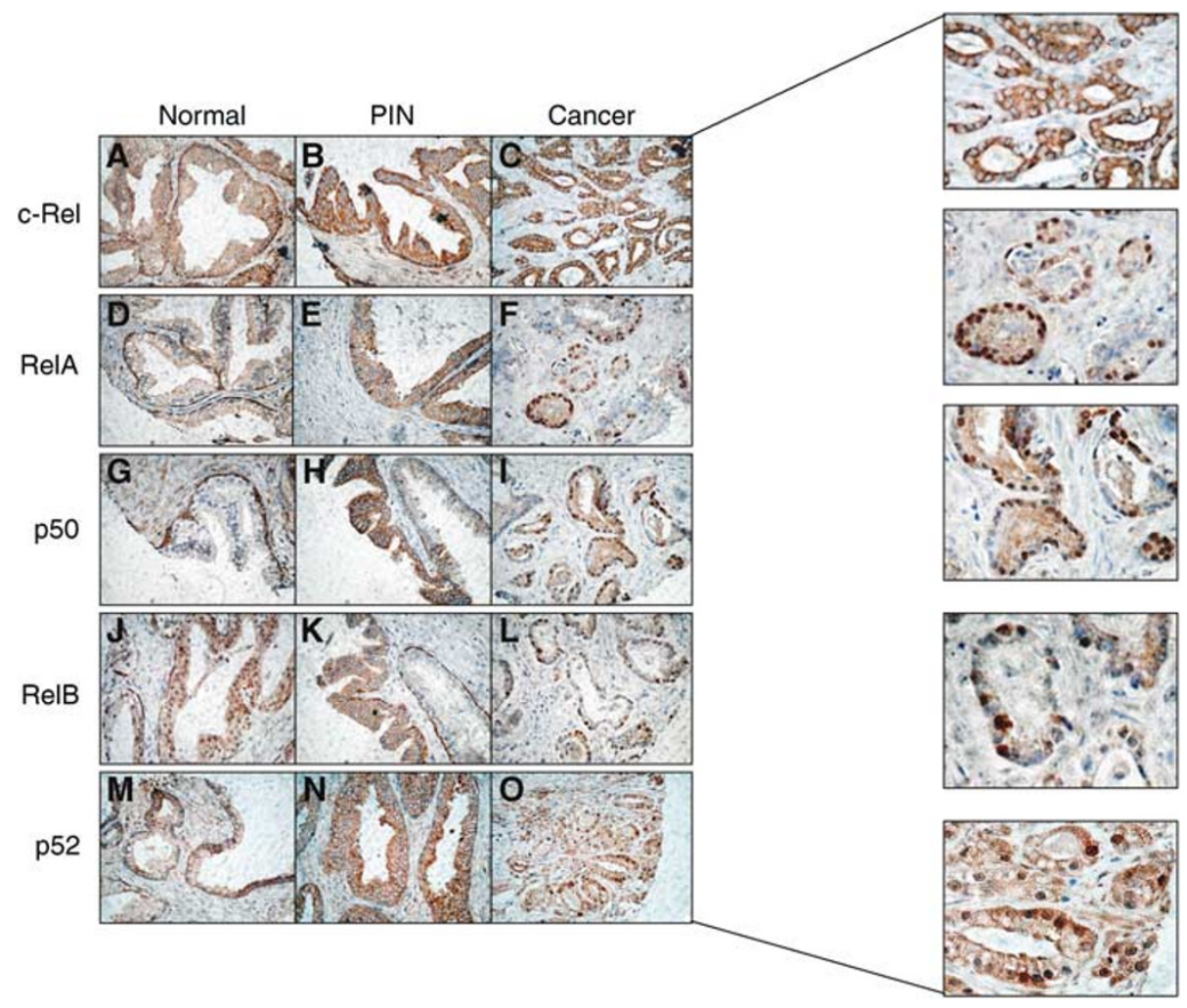

Figure I Immunohistochemical detection of c-Rel $(\mathbf{A}-\mathbf{C})$, RelA (D-F), p50 (G-I), RelB (J-L), and p52 (M-O) in normal, PIN, and cancerous prostate tissues $(\times 400)$. Note nuclear RelB staining in a subset of normal glands $(\mathbf{J})$. Also note a mixture of weak and strong p52 cytoplasmic staining in normal glands (M). Enlargements are provided to more clearly distinguish the subcellular localisation in cancer specimens.

Table I Nuclear localisation of NF- $\kappa$ B transcription factors in prostatic tissue cores

\begin{tabular}{|c|c|c|c|c|c|c|c|c|c|c|c|c|}
\hline Tissue type & \multicolumn{3}{|c|}{ RelA } & \multicolumn{3}{|c|}{ p50 } & \multicolumn{3}{|c|}{ RelB } & \multicolumn{3}{|c|}{ p52 } \\
\hline Normal & 39 & $0(0)$ & & 37 & $0(0)$ & & 40 & $2(5.0)$ & & 39 & I (2.6) & \\
\hline PIN & 23 & I (4.3) & & 21 & $0(0)$ & & 24 & $0(0)$ & & 24 & $0(0)$ & \\
\hline Cancer & 282 & $44(15.6)$ & 0.001 & 285 & $30(10.5)$ & 0.004 & 282 & $75(26.6)$ & $<0.001$ & 281 & $30(10.7)$ & 0.025 \\
\hline
\end{tabular}

$\chi^{2}$ was used to test the association of nuclear NF- $\kappa$ B expression with tissue type (cancer vs noncancer). $P$-value less than 0.05 was considered as significant. NF- $\kappa \mathrm{B}=$ nuclear factor- $\kappa \mathrm{B} ; \mathrm{PIN}=$ prostatic intraepithelial neoplasia.

Table 2 Nuclear localisation of NF- $\kappa$ B transcription factors in low to intermediate $(2,2 / 3,3)$ and high $(3 / 4,4,4 / 5,5)$ Gleason grade scores

\begin{tabular}{|c|c|c|c|c|c|c|c|c|c|c|c|c|}
\hline \multirow[b]{2}{*}{ Gleason group } & \multicolumn{3}{|c|}{ RelA } & \multicolumn{3}{|c|}{ p50 } & \multicolumn{3}{|c|}{ RelB } & \multicolumn{3}{|c|}{ p52 } \\
\hline & $\mathbf{N}$ & $\begin{array}{c}\text { No. } \\
\text { positive (\%) }\end{array}$ & $P$ & $N$ & $\begin{array}{c}\text { No. } \\
\text { positive (\%) }\end{array}$ & $P$ & $N$ & $\begin{array}{c}\text { No. } \\
\text { positive (\%) }\end{array}$ & $P$ & $\mathbf{N}$ & $\begin{array}{c}\text { No. } \\
\text { positive (\%) }\end{array}$ & $P$ \\
\hline Low & 170 & $24(14.1)$ & & 174 & $16(9.2)$ & & 169 & $40(23.7)$ & & 167 & $16(9.6)$ & \\
\hline High & 112 & $20(17.9)$ & 0.405 & 111 & $14(12.6)$ & 0.431 & 113 & $35(31.0)$ & 0.104 & 114 & $14(12.3)$ & 0.555 \\
\hline
\end{tabular}

$\chi^{2}$ was used to test the association between nuclear NF- $\kappa$ B and tissue grade. P-value less than 0.05 was considered as significant. NF- $\kappa$ B $=$ nuclear factor- $\kappa$ B.

levels, pathological stage, and patient's Gleason score. No significant relationship was obtained between nuclear NF- $\kappa \mathrm{B}$ and pPSA or pathological stage. In contrast, the number of nuclear RelB-positive cores increased significantly with increasing Gleason scores (Spearman coefficient: $0.167 ; P=0.018$; Table 3).

\section{DISCUSSION}

Although prostate cancer can be detected in its early stages, it is still difficult to predict whether it will remain latent or progress to an advanced, metastatic disease. This is largely due to the lack of 


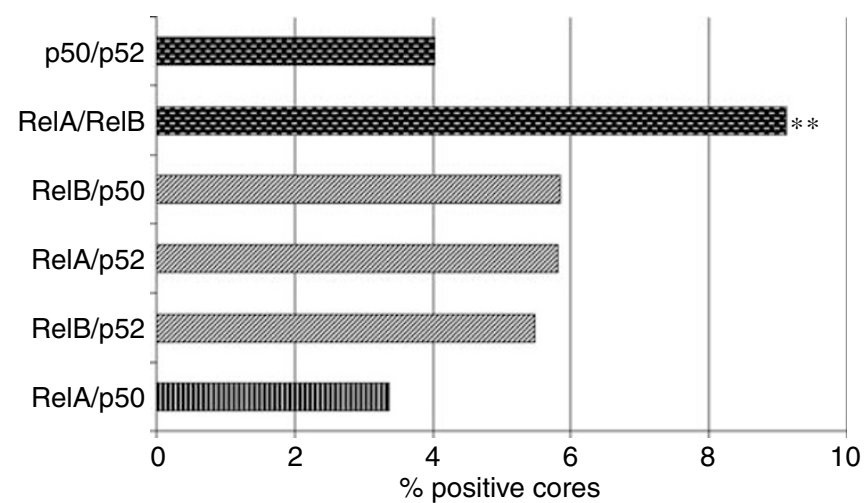

Figure 2 Frequencies of $\mathrm{NF}-\kappa \mathrm{B}$ subunit combinations in the prostate cancer cores. The vertical pattern is for the canonical RelA-p50 pair. Noncanonical combinations are represented as follows: the diagonal pattern is for other class I/class 2 dimers, and the dashed pattern is for class I/class I or class $2 /$ class 2 dimers. $* * P<0.01 \chi^{2}$ between RelA-p50 and RelA-RelB.

Table 3 Correlation between NF- $\kappa$ B nuclear localisation and patient's Gleason scores

\begin{tabular}{lcl}
\hline Subunits & Coefficient & P-value \\
\hline RelA & 0.078 & 0.272 \\
p50 & -0.018 & 0.803 \\
RelB & 0.167 & $0.018^{*}$ \\
p52 & -0.019 & 0.793 \\
\hline
\end{tabular}

Spearman's $\sigma$ coefficient was used to test the relationship between nuclear NF- $\kappa \mathrm{B}$ expression and Gleason scores. ${ }^{*} P$-value less than 0.05 was considered as significant. $\mathrm{NF}-\kappa \mathrm{B}=$ nuclear factor $-\kappa \mathrm{B}$

clinically proven molecular markers of prostate cancer progression. There are hundreds of candidate genes and proteins under investigation but few have been validated in multivariate analyses (Tricoli et al, 2004). We and others have recently shown that RelA, a member of the NF- $\kappa \mathrm{B}$ transcription factor family, is an independent predictor of biochemical recurrence (Ayala et al, 2004; Fradet et al, 2004; Ross et al, 2004). In addition, we have detected high levels of nuclear RelA staining in lymph node metastases and in patients who developed bone metastases (Lessard et al, 2003; Ismail et al, 2004). Hence, RelA appears to play a role in the progression of prostate cancer but little is known about the status of other NF- $\kappa \mathrm{B}$ family members, and no simultaneous analysis of the subcellular localisation of these subunits has been previously reported in prostate tissues.

In the present study, we characterised the expression and the subcellular localisation of RelA, p50, RelB, p52, and c-Rel. All subunits are expressed in prostatic tissues, and all but c-Rel can be detected in the nucleus of prostatic cells, more frequently in cancerous tissues. This suggests that both canonical (RelA, p50) and the noncanonical (e.g., RelB, p52) NF- $\kappa$ B subunits can be activated in prostate cancer cells, whereas c-Rel remains inactive. Notably, a recent study reported a negative or nonspecific p50 expression in prostate tumours (Shukla et al, 2004). Conversely, we observed high levels of cytoplasmic p50 expression in cancer tissues. This discrepancy may be related to use of different antibodies.

The NF- $\kappa \mathrm{B}$ subunits can potentially form six heterodimers: RelA/p50, RelB/p52, RelA/p52, RelB/p50, RelA/RelB, and p50/p52. Importantly, each dimer may differently affect the cellular transcriptome, thus influencing disease progression. While in this study we did not directly assess the presence of specific heterodimers in prostate cancer specimens, our results provide an indication of those subunits present in the nucleus of cancer cells. Our results clearly demonstrate that within a given sample, we were more likely to find nuclear RelA and RelB, while the nuclear localisation of RelA and p50 was less frequently observed. If these subunits are relocalised within the same cell, this would suggest that noncanonical dimers are more frequently activated in prostate cancer, which is in contrast with the more usually observed activation of the canonical pathway in other cancers. Since RelA - RelB dimers are not regulated by $\mathrm{I} \kappa \mathrm{B}$ proteins, they may act together or in concert with other transcription factors to attract histone acetyl transferases and cofactors and constitutively drive the transcription of specific genes. On the other hand, it has been reported that RelB forms transcriptionally inactive complexes with RelA in mouse embryonic fibroblasts (Marienfeld et al, 2003). In this model, RelB sequesters RelA and thereby represses RelAdependent transcription. Whether RelA-RelB complexes exist in prostate cancer cells remains to be elucidated, but our results justify further investigation.

There is also a possibility that the RelB-p52 combination is more frequent than the canonical RelA - p50 pair (Figure 2). Given that RelB and p52 have been shown to be activated in other tumour types such as B- and T-cell lymphomas as well as in breast carcinoma (Dejardin et al, 1998; Rayet and Gelinas, 1999; Cogswell et al, 2000), they may also play a role in prostate cancer development and progression. Interestingly, both nuclear RelB and nuclear p52 were also detected in a subset of benign tissues (Table 1). However, it is important to note that all benign tissues collected were from cancerous prostates. Consequently, the nuclear localisation of RelB and p52 may evoke a normal transient activation, but may also reflect a cellular response to the tumour environment or a transitional state towards transformation. To test this hypothesis, we are presently characterising the activation status of NF- $\kappa \mathrm{B}$ subunits in cancer-free prostate specimens obtained from young male autopsies.

Correlation analyses between NF- $\kappa \mathrm{B}$ subunits and relevant clinical parameters revealed a significant relationship between RelB nuclear expression and patient's Gleason score (Table 3). Similarly, there was also a trend towards higher RelB nuclear staining in high-grade cancer tissue cores (Table 2). Whether RelB or other subunits confer a more aggressive phenotype to prostatic carcinoma remains to be determined. To this end, we are presently expanding the analysis to more clearly define the role of RelB in prostate cancer progression.

The absence of a correlation between RelA nuclear expression and patient's Gleason score confirms our previous observations (Lessard et al, 2003). Despite this, RelA acts as an independent predictor of patient's outcome (Fradet et al, 2004), which suggests that p50 and p52 should also be analysed to assess their potential as prognostic markers.

To our knowledge, this is the first large-scale immunohistochemical characterisation of NF- $\kappa \mathrm{B}$ transcription factors in the same cohort of prostate cancer patients. Our results not only provide further evidence for a role of the canonical NF- $\kappa \mathrm{B}$ pathway in prostate cancer, but also suggest a potential role for other $\mathrm{NF}-\kappa \mathrm{B}$ subunits and pathways. Studies are ongoing to validate the usefulness of specific NF- $\kappa \mathrm{B}$ subunits, or combinations, as prognostic markers.

\section{ACKNOWLEDGEMENTS}

This work was supported by a grant from The Cancer Research Society of Canada. FS holds the University of Montreal Chair in Prostate Cancer Research. A-M M-M is a recipient of a Chercheur National fellowship from the Fonds de la Recherche en Santé du Québec (FRSQ). LL is supported by a Canadian Institute of Health Research/Canadian Prostate Cancer Research Initiative studentship. We thank Cécile Le Page, PhD, and Jean-Simon Diallo for their critical review of the manuscript and David Iannuzzi for statistical assistance. 


\section{REFERENCES}

Aggarwal BB (2004) Nuclear factor-kappaB: the enemy within. Cancer Cell 6: $203-208$

Ayala GE, Dai H, Ittmann M, Li R, Powell M, Frolov A, Wheeler TM, Thompson TC, Rowley D (2004) Growth and survival mechanisms associated with perineural invasion in prostate cancer. Cancer Res 64: $6082-6090$

Baldwin AS (2001) Control of oncogenesis and cancer therapy resistance by the transcription factor NF-kappaB. J Clin Invest 107: $241-246$

Barkett M, Gilmore TD (1999) Control of apoptosis by Rel/NF-kappaB transcription factors. Oncogene 18: 6910-6924

Chen CD, Sawyers CL (2002) NF-kappa B activates prostate-specific antigen expression and is upregulated in androgen-independent prostate cancer. Mol Cell Biol 22: 2862-2870

Cogswell PC, Guttridge DC, Funkhouser WK, Baldwin Jr AS (2000) Selective activation of NF-kappa B subunits in human breast cancer: potential roles for NF-kappa $\mathrm{B} 2 / \mathrm{p} 52$ and for $\mathrm{Bcl}-3$. Oncogene 19: $1123-1131$

Dejardin E, Deregowski V, Greimers R, Cai Z, Chouaib S, Merville MP, Bours V (1998) Regulation of major histocompatibility complex class I expression by NF-kappaB-related proteins in breast cancer cells. Oncogene 16: 3299-3307

Fradet V, Lessard L, Begin LR, Karakiewicz P, Masson AM, Saad F (2004) Nuclear factor-kappaB nuclear localization is predictive of biochemical recurrence in patients with positive margin prostate cancer. Clin Cancer Res 10: 8460 - 8464

Gasparian AV, Yao YJ, Kowalczyk D, Lyakh LA, Karseladze A, Slaga TJ, Budunova IV (2002) The role of IKK in constitutive activation of NF-kappaB transcription factor in prostate carcinoma cells. J Cell Sci 115: $141-151$

Ghosh S, Karin M (2002) Missing pieces in the NF-kappaB puzzle. Cell 109(Suppl): S81 - S96

Hodge JC, Bub J, Kaul S, Kajdacsy-Balla A, Lindholm PF (2003) Requirement of RhoA activity for increased nuclear factor kappaB activity and PC-3 human prostate cancer cell invasion. Cancer Res 63: $1359-1364$

Huang S, Pettaway CA, Uehara H, Bucana CD, Fidler IJ (2001) Blockade of NF-kappaB activity in human prostate cancer cells is associated with suppression of angiogenesis, invasion, and metastasis. Oncogene 20: $4188-4197$

Ismail HA, Lessard L, Mes-Masson AM, Saad F (2004) Expression of NF-kappaB in prostate cancer lymph node metastases. Prostate 58: $308-313$

Lessard L, Mes-Masson AM, Lamarre L, Wall L, Lattouf JB, Saad F (2003) NF-kappa B nuclear localization and its prognostic significance in prostate cancer. BJU Int 91: $417-420$
Levine L, Lucci III JA, Pazdrak B, Cheng JZ, Guo YS, Townsend Jr CM, Hellmich MR (2003) Bombesin stimulates nuclear factor kappa B activation and expression of proangiogenic factors in prostate cancer cells. Cancer Res 63: 3495-3502

Ling MT, Wang X, Ouyang XS, Xu K, Tsao SW, Wong YC (2003) Id-1 expression promotes cell survival through activation of NF-kappaB signalling pathway in prostate cancer cells. Oncogene 22: 4498-4508

Liptay S, Seriu T, Bartram CR, Schmid RM (1997) Germline configuration of nfkb2, c-rel and bcl3 in childhood acute lymphoblastic leukemia (ALL). Leukemia 11: $1364-1366$

Marienfeld R, May MJ, Berberich I, Serfling E, Ghosh S, Neumann M (2003) RelB forms transcriptionally inactive complexes with RelA/p65. J Biol Chem 278: $19852-19860$

Munzert G, Kreitmeier S, Bergmann L (2000) Normal structure of NFKB2, $\mathrm{C}-\mathrm{REL}$ and BCL-3 gene loci in lymphoproliferative and myeloproliferative disorders. Leuk Lymphoma 38: 395-400

Rayet B, Gelinas C (1999) Aberrant rel/nfkb genes and activity in human cancer. Oncogene 18: 6938-6947

Ross JS, Kallakury BV, Sheehan CE, Fisher HA, Kaufman Jr RP, Kaur P, Gray K, Stringer B (2004) Expression of nuclear factor-kappa B and I kappa $\mathrm{B}$ alpha proteins in prostatic adenocarcinomas: correlation of nuclear factor-kappa B immunoreactivity with disease recurrence. Clin Cancer Res 10: 2466-2472

Shukla S, MacLennan GT, Fu P, Patel J, Marengo SR, Resnick MI, Gupta S (2004) Nuclear factor-kappaB/p65 (Rel A) is constitutively activated in human prostate adenocarcinoma and correlates with disease progression. Neoplasia 6: $390-400$

Sovak MA, Bellas RE, Kim DW, Zanieski GJ, Rogers AE, Traish AM, Sonenshein GE (1997) Aberrant nuclear factor-kappaB/Rel expression and the pathogenesis of breast cancer. J Clin Invest 100: 2952-2960

Suh J, Payvandi F, Edelstein LC, Amenta PS, Zong WX, Gelinas C, Rabson AB (2002) Mechanisms of constitutive NF-kappaB activation in human prostate cancer cells. Prostate 52: $183-200$

Sweeney C, Li L, Shanmugam R, Bhat-Nakshatri P, Jayaprakasan V, Baldridge LA, Gardner T, Smith M, Nakshatri H, Cheng L (2004) Nuclear factor-kappaB is constitutively activated in prostate cancer in vitro and is overexpressed in prostatic intraepithelial neoplasia and adenocarcinoma of the prostate. Clin Cancer Res 10: 5501-5507

Tricoli JV, Schoenfeldt M, Conley BA (2004) Detection of prostate cancer and predicting progression: current and future diagnostic markers. Clin Cancer Res 10: 3943 - 3953

Wang W, Abbruzzese JL, Evans DB, Larry L, Cleary KR, Chiao PJ (1999) The nuclear factor-kappa B RelA transcription factor is constitutively activated in human pancreatic adenocarcinoma cells. Clin Cancer Res 5: $119-127$ 